\title{
SOME ASPECTS OF THE GEOMETRIC STRUCTURE OF THE SMOOTH DUAL OF $p$-ADIC REDUCTIVE GROUPS
}

\author{
ANNE-MARIE AUBERT \\ CNRS, Sorbonne Université, Université de Paris \\ Institut de Mathématiques de Jussieu - Paris Rive Gauche \\ F-75005 Paris, France \\ ORCID: 0000-0002-9613-9140Ｅ-mail: anne-marie.aubert@imj-prg.fr
}

\begin{abstract}
We review the ABPS Conjecture in the case of a split $p$-adic reductive group $G$, its links with the Langlands correspondence for $G$, and how it can be used in order to provide a conjectural description for $K_{*}\left(C_{\text {red }}^{*}(G)\right)$.
\end{abstract}

1. Introduction and a few memories. The first time I heard the name of Paul Baum was in a talk given by Vincent Lafforgue at the École Normale Supérieure on the "BaumConnes conjecture" in the end of the 90's. A few years later, while I was already working with Roger Plymen, I had the opportunity to start collaborating with Paul also. A series of exchanges of emails and files with Paul and Roger led to two papers [ABP1, [ABP2] on the very early stages of what is now known as the "ABPS conjecture". A version of the conjecture, that was stated and studied jointly with Paul, Roger and Maarten Solleveld, is recalled in Section 3

I met Paul for the first time at the Institut Henri Poincaré (IHP) in Paris, on his way to visiting the Université Clermont Auvergne. I had a very interesting mathematical discussion with Paul, and he talked also in French with my Mother and told her that he travelled several times with his own Mother to mathematical conferences.

We met again at several occasions, notably at three "birthday conferences": the one in honor of Alain Connes in 2007 in Paris, the one in honor of Roger the year after in Manchester, and the "Conference on Geometry, Representation Theory and the BaumConnes Conjecture" in 2016 at the Fields Institute in Toronto, whose aim was also to celebrate the 80th birthday of Paul. I was very glad and honored in giving a talk at

2010 Mathematics Subject Classification: Primary 20C08; Secondary 14F43, 20G20.

The paper is in final form and no version of it will be published elsewhere. 
the conference on joint works with Paul, Maarten, Roger and my former student Ahmed Moussaoui, which link the Baum-Connes conjecture to the Langlands program. Paul himself gave a beautiful talk on our work ABPS10, the most recent of a long list of joint papers [ABPS1, ABPS2, ABPS3, ABPS4, ABPS5, ABPS6], ABPS7, ABPS8], ABPS9. I enjoy always very much attending Paul's talks: I really appreciate his ability in presenting the key ideas of a rather technical subject in a very simple and limpid manner. For more memories, the reader is invited to look at [APS].

Here is a brief outline of the article. In the first section we will describe two kinds of extended quotients that we will call geometric and spectral, respectively. The notion of geometric (also called "ordinary") extended quotient of a complex affine variety by a finite group $\Gamma$ acting on it was brought by Paul from the world of noncommutative geometry to that of representations of $p$-adic groups. The spectral extended quotient is the analogue of the latter in which the role of the conjugacy classes of $\Gamma$ is played by the isomorphism classes of irreducible representations of $\Gamma$.

In the rest of the article, we will focus on the spectral version and explain how it occurs in the theory of smooth representations of split $p$-adic reductive groups and in the Langlands program via the ABPS conjecture and a Galois version of the latter (that is in fact a theorem: Theorem 5.1, respectively. The key ingredients are the decompositions into Bernstein series of the set of isomorphism classes of irreducible smooth representations of the $p$-adic group (due to Bernstein) and of the set of its enhanced Langlands parameters (obtained in AMS1]). In the last section, we will explain the relation of the Bernstein decomposition of the reduced $C^{*}$-algebra of a split $p$-adic group $G$ with the Baum-Connes Conjecture for $G$ (which was proved by V. Lafforgue).

The results presented in the article are specializations to the case of split groups of more elaborated versions (see in particular [ABPS8, and AMS1) that involve certain non-trivial twists in general, and notably a notion of twisted extended quotient.

2. The geometric and spectral extended quotients. Let $\Gamma$ be a finite group acting as automorphisms of a complex affine variety $X$. For $x \in X$, let $\Gamma_{x}$ denote the stabilizer group of $x$ :

$$
\Gamma_{x}:=\{\gamma \in \Gamma: \gamma \cdot x=x\} .
$$

We denote by $\underline{\Gamma}_{x}$ the set of conjugacy classes of $\Gamma_{x}$ and by $\operatorname{Irr}\left(\Gamma_{x}\right)$ the set of equivalence classes of irreducible representations of $\Gamma_{x}$.

We set

$$
\begin{aligned}
\widetilde{X}_{\text {geo }} & :=\left\{(x, \underline{\gamma}) \in X \times \Gamma: \underline{\gamma} \in \underline{\Gamma}_{x}\right\}, \\
\widetilde{X}_{\text {spec }} & :=\left\{(x, \tau) \in X \times \operatorname{Irr}(\Gamma): \tau \in \operatorname{Irr}\left(\Gamma_{x}\right)\right\} .
\end{aligned}
$$

Then $\Gamma$ acts on $\tilde{X}_{\text {geo }}$ by

$$
\gamma^{\prime} \cdot(x, \underline{\gamma}):=\left(\gamma^{\prime} \cdot x, \gamma^{\prime} \underline{\gamma}\left(\gamma^{\prime}\right)^{-1}\right)
$$

on $\widetilde{X}_{\text {spec }}$ by

$$
\gamma^{\prime} \cdot(x, \tau):=\left(\gamma^{\prime} \cdot x, \gamma_{*}^{\prime} \tau\right),
$$

where $\gamma_{*}^{\prime}: \operatorname{Irr}\left(\Gamma_{x}\right) \rightarrow \operatorname{Irr}\left(\Gamma_{\gamma^{\prime} x}\right)$. 
Definition 2.1. We define the geometric and spectral extended quotients of $X$ by $\Gamma$ as

$$
X / / \underline{\Gamma}:=\tilde{X}_{\text {geo }} / \Gamma \text { and } X / / \Gamma:=\widetilde{X}_{\text {spec }} / \Gamma
$$

respectively.

The quotient variety $X / \Gamma$ is obtained by collapsing each orbit to a point, it is an affine variety. The geometric extended quotient is obtained by replacing the orbit of $x \in X$ by $\underline{\Gamma}_{x}$, while the spectral extended quotient is obtained by replacing it by the set $\operatorname{Irr}\left(\Gamma_{x}\right)$.

REMARK 2.2. For each $x \in X$, the sets $\underline{\Gamma}_{x}$ and $\operatorname{Irr}\left(\Gamma_{x}\right)$ are in bijection, but not in canonical way in general. It follows that the extended quotients $X / / \underline{\Gamma}$ and $X / / \Gamma$ are in bijection, but again not in canonical way in general.

The geometric extended quotient $X / / \underline{\Gamma}$, also called the ordinary extended quotien $\left.\right|^{1}$, coincides with the transformation groupoid attached to the $\Gamma$-set $X$. Recall that the latter is the small category with objects the elements of $X$ and with morphisms $x \rightarrow y$ the pairs $(x, \gamma)$ such that $\gamma \cdot x=y$, the composition being defined as $\left(x^{\prime}, \gamma^{\prime}\right) \circ(x, \gamma):=\left(x, \gamma^{\prime} \gamma\right)$, which is defined provided $x^{\prime}=\gamma \cdot x$.

The projections $(x, 0) \mapsto x$ and $(x, \tau) \mapsto x$ from $\widetilde{X}_{\text {geo }}$ and $\widetilde{X}_{\text {spec }}$ to $X$ are $\Gamma$-equivariant and so pass to quotient spaces to give morphisms of affine varieties

$$
\mathrm{pr}_{\mathrm{geo}}: X / / \underline{\Gamma} \rightarrow X / \Gamma \text { and } \mathrm{pr}_{\mathrm{spec}}: X / / \Gamma \rightarrow X / \Gamma
$$

These maps will be referred to as the projections of the extended quotients onto the ordinary quotient.

The inclusions $x \mapsto(x, e)$ and $x \mapsto(x$, triv $)$ of $X$ into $\widetilde{X}_{\text {geo }}$ and $\widetilde{X}_{\text {spec }}$ are $\Gamma$-equivariant and so pass to quotient spaces to give inclusions of affine varieties

$$
X / \Gamma \hookrightarrow X / / \underline{\Gamma} \text { and } X / \Gamma \hookrightarrow X / / \Gamma \text {. }
$$

with $X / \Gamma$ removed will be denoted $X / / \underline{\Gamma}-X / \Gamma$ and $X / / \Gamma-X / \Gamma$, respectively.

3. Extended quotients and representations of $p$-adic groups: the ABPS conjecture. Let $F$ be a local non-Archimedean field with finite residual field $k_{F}=\mathbb{F}_{q}$. Let $F_{\text {sep }}$ be a fixed separable closure of $F$, and let $\Gamma_{F}$ denote the Galois group of $F_{\text {sep }} / F$. Let $W_{F}$ denote the Weil group $W_{F}$ of $F$ (relative to $F_{\text {sep }}$ ), and let $I_{F} \subset W_{F}$ be the inertia subgroup.

Let $\mathbf{G}$ be a connected reductive algebraic group defined and split over $F$, and let $G$ denote the group of the $F$-rational points of $\mathbf{G}$.

3.1. The Bernstein center. Let $\operatorname{Rep}(G)$ denote the category of smooth representations of $G$. Recall that an irreducible smooth complex representation of the group $G$ is called supercuspidal if it does not appear in any $G$-representation induced from a proper Levi subgroup of $G$. An irreducible $G$-representation is supercuspidal if and only if all its matrix coefficients have compact support modulo the center of $G$.

\footnotetext{
${ }^{1}$ In this terminology, the spectral extended quotient is called the extended quotient of the second kind.
} 
Let $P$ be a parabolic subgroup of $G$ with Levi factor $L$ and write $\mathrm{I}_{P}^{G}$ for the functor of normalized parabolic induction from $\operatorname{Rep}(L)$ to $\operatorname{Rep}(G)$. Let $\sigma$ be a supercuspidal irreducible representation of $L$. We call $(L, \sigma)$ a cuspidal pair, and we consider such pairs up to inertial equivalence: this is the equivalence relation generated by

- unramified twists, $(L, \sigma) \sim(L, \sigma \otimes \chi)$ for $\chi \in X_{\mathrm{nr}}(L)$, where $X_{\mathrm{nr}}(L)$ is the group of unramified (not necessarily unitary) characters $L \rightarrow \mathbb{C}^{\times}$;

- $G$-conjugation, $(L, \sigma) \sim\left(g L g^{-1}, g \cdot \sigma\right)$ for $g \in G$.

We denote a typical inertial equivalence class by $\mathfrak{s}=[L, \sigma]_{G}$. In particular

$$
\mathfrak{s}_{L}:=[L, \sigma]_{L}=\left\{\sigma \otimes \chi \in \operatorname{Irr}(L): \chi \in X_{\mathrm{nr}}(L)\right\} .
$$

If $\pi \in \operatorname{Irr}(G)$ is a constituent of $\mathrm{I}_{P}^{G}(\tau)$ for some $\tau \in \mathfrak{s}_{L}$, then $\mathfrak{s}_{L}$ is called the supercuspidal support of $\pi$. Bernstein attached to every $\mathfrak{s}$ a block in the category $\operatorname{Rep}(G)$, in the following way. We define

$$
\operatorname{Irr}(G)^{\mathfrak{s}}=\left\{\pi \in \operatorname{Irr}(G): \pi \text { has supercuspidal support } \mathfrak{s}_{L}\right\},
$$

$\operatorname{Rep}(G)^{\mathfrak{s}}=\left\{\pi \in \operatorname{Rep}(G)\right.$ : every irreducible constituent of $\pi$ belongs to $\left.\operatorname{Irr}(G)^{\mathfrak{s}}\right\}$.

We denote the set of all inertial equivalence classes for $G$ by $\mathfrak{B}(G)$.

The category $\operatorname{Rep}(G)$ decomposes as

$$
\operatorname{Rep}(G)=\prod_{\mathfrak{s} \in \mathfrak{B}(G)} \operatorname{Rep}(G)^{\mathfrak{s}},
$$

see [BeDe, Proposition 2.10]. In particular, the space of irreducible $G$-representations is a disjoint union

$$
\operatorname{Irr}(G)=\bigsqcup_{\mathfrak{s} \in \mathfrak{B}(G)} \operatorname{Irr}(G)^{\mathfrak{s}} .
$$

Let $\operatorname{Irr}_{\text {cusp }}(L)$ be the set of isomorphism classes of supercuspidal irreducible smooth representations of $L$. For $\sigma \in \operatorname{Irr}_{\text {cusp }}(L)$ (and in fact for every irreducible $L$-representation) the group

$$
X_{\mathrm{nr}}(L, \sigma):=\left\{\chi \in X_{\mathrm{nr}}(L): \sigma \otimes \chi \cong \sigma\right\}
$$

is finite. Thus there is a bijection

$$
X_{\mathrm{nr}}(L) / X_{\mathrm{nr}}(L, \sigma) \rightarrow \operatorname{Irr}(L)^{\mathfrak{s}_{L}}: \chi \mapsto \sigma \otimes \chi,
$$

which endows $\operatorname{Irr}(L)^{\mathfrak{s}_{L}}$ with the structure of a complex torus. Up to isomorphism this torus depends only on $\mathfrak{s}$, and it is known as the Bernstein torus $T_{\mathfrak{s}}$ attached to $\mathfrak{s}$. We note that $T_{\mathfrak{s}}$ is only an algebraic variety, it is not endowed with a natural multiplication map. The group $W(G, L):=\mathrm{N}_{G}(L) / L$ acts on $\operatorname{Irr}(L)$ by

$$
w \cdot \pi=\left[\bar{w} \cdot \pi: l \mapsto \pi\left(\bar{w}^{-1} l \bar{w}\right)\right] \quad \text { for any lift } \bar{w} \in \mathrm{N}_{G}(L) \text { of } w \in W(G, L) .
$$

Bernstein also associated to every $\mathfrak{s}$ the finite group

$$
W_{\mathfrak{s}}:=\left\{w \in W(G, L): w \cdot \operatorname{Irr}(L)^{\mathfrak{s}_{L}}=\operatorname{Irr}(L)^{\mathfrak{s}_{L}}\right\} .
$$

It acts naturally on $T_{\mathfrak{s}}$, by automorphisms of algebraic varieties.

By [BeDe, Théorème 2.13] the categorical center of the Bernstein block $\operatorname{Rep}^{\mathfrak{s}}(G)$ is

$$
\mathrm{Z}\left(\operatorname{Rep}(G)^{\mathfrak{s}}\right) \cong \mathcal{O}\left(T_{\mathfrak{s}}\right)^{W_{\mathfrak{s}}}=\mathcal{O}\left(T_{\mathfrak{s}} / W_{\mathfrak{s}}\right) .
$$


Here $\mathcal{O}$ stands for the regular functions on an affine variety. Then taking supercuspidal support gives a map

$$
\text { sc }: \operatorname{Irr}(G)^{\mathfrak{s}} \rightarrow T_{\mathfrak{s}} / W_{\mathfrak{s}}
$$

which is surjective and has finite fibers BeDe, §3]. Every $\pi \in \operatorname{Irr}(G)$ is a constituent of $I_{P}^{G}(\sigma)$, where $[L, \sigma]_{G}$ is uniquely determined. By (4) the supercuspidal $L$-representation $\sigma \in T_{\mathfrak{s}}$ is in fact uniquely determined up to $W_{\mathfrak{s}}$. The map $\pi \mapsto W_{\mathfrak{s}} \sigma$ is just sc, and for this reason it is called the cuspidal support map. Via this map $\operatorname{Irr}^{\mathfrak{s}}(G)$ can be regarded as a non-separated algebraic variety lying over $T_{\mathfrak{s}} / W_{\mathfrak{s}}$.

3.2. The statement of the conjecture. Let $\mathfrak{s}=[L, \sigma]_{G}$ be an inertial equivalence class for $G$. Let $W_{\mathfrak{s}, t}$ be the stabilizer in $W_{\mathfrak{s}}$ of a point $t \in T_{\mathfrak{s}}$.

The ABPS conjecture from [ABPS1, §15] and [ABPS8, Conjecture 2] in its roughest form asserts that there exists a bijection

$$
\operatorname{Irr}(G)^{\mathfrak{s}} \longleftrightarrow T_{\mathfrak{s}} / / W_{\mathfrak{s}}
$$

such that:

(a) it restricts to a bijection between tempered representations and the unitary part of the extended quotient (as explained below);

(b) it is canonical up to permutations within $L$-packets $\Pi_{\phi}(G)$ (see Section 4), that is, for any Langlands parameter $\phi$ for $G$, the image of $\Pi_{\phi}(G) \cap \operatorname{Irr}^{\mathfrak{s}}(G)$ is canonically defined (assuming the existence of the LLC for $G$ ).

The definitions of $W_{\mathfrak{s}}$ and of extended quotients imply that for a fixed Levi subgroup $L$ of $G$ there is a natural bijection

$$
\bigsqcup_{\mathfrak{s}=[L, \sigma]_{G}} T_{\mathfrak{s}} / / W_{\mathfrak{s}} \rightarrow \operatorname{Irr}_{\text {cusp }}(L) / / W(G, L) .
$$

Let $\mathcal{L}(G)$ be a set of representatives for the $G$-conjugacy classes of Levi subgroups of $G$. The ABPS conjecture can also be formulated in terms of a bijection

$$
\operatorname{Irr}(G) \longleftrightarrow \bigsqcup_{L \in \mathcal{L}(G)} \operatorname{Irr}_{\text {cusp }}(L) / / W(G, L)
$$

In this version, the conjecture asserts that $\operatorname{Irr}(G)$ is determined by a much smaller set of data, namely the supercuspidal representations of Levi subgroups $L$ of $G$, and the actions of the Weyl groups $W(G, L)$ on those.

Recall [Wal, §III.1-III.2] that a supercuspidal representation is tempered if and only if it is unitary.

Definition 3.1. Let $T_{\mathfrak{s} \text {, un }}$ be the set of unitary representations in $T_{\mathfrak{s}}$, a $W_{\mathfrak{s}}$-stable compact real subtorus.

Let $X_{\text {unr }}(L)$ denote the group of unitary unramified characters of $L$. Without loss of generality we may assume that the basepoint $\sigma \in T_{\mathfrak{s}}$ is unitary. Then (1) becomes a bijection

$$
X_{\mathrm{unr}}(L) / X_{\mathrm{unr}}(L, \sigma) \rightarrow T_{\mathfrak{s}, \mathrm{un}}: \chi \mapsto \sigma \otimes \chi
$$


Let $X_{\mathrm{nr}}^{+}(L)$ be the group of unramified characters $L \rightarrow \mathbb{R}_{>0}$. The polar decomposition of $X_{\mathrm{nr}}(L)$ reads

$$
X_{\mathrm{nr}}(L)=X_{\mathrm{unr}}(L) \times X_{\mathrm{nr}}^{+}(L) .
$$

Since $X_{\mathrm{nr}}(L, \sigma)$ is finite and $\mathbb{R}_{>0}$ has no nontrivial finite subgroups, $X_{\mathrm{nr}}(L, \sigma) \cap$ $X_{\mathrm{nr}}^{+}(L)=\{1\}$. Hence the canonical map

$$
T_{\mathfrak{s}, \text { un }} \times X_{\mathrm{nr}}^{+}(L) \rightarrow T_{\mathfrak{s}}:\left(\sigma, \chi^{+}\right) \mapsto \sigma \otimes \chi^{+}
$$

is bijective. We regard $(9)$ as the polar decomposition of $T_{\mathfrak{s}}$.

Let $\operatorname{Irr}_{\mathrm{t}}(G)$ be the set of irreducible tempered $G$-representations (still considered up to isomorphism) and write

$$
\operatorname{Irr}_{\mathrm{t}}(G)^{\mathfrak{s}}=\operatorname{Irr}(G)^{\mathfrak{s}} \cap \operatorname{Irr}_{\mathrm{t}}(G) .
$$

Part (a) in the ABPS conjecture asserts that there is a bijection

$$
\operatorname{Irr}_{\mathrm{t}}(G)^{\mathfrak{s}} \longleftrightarrow T_{\mathfrak{s}, \text { un }} / / W_{\mathfrak{s}} \text {. }
$$

In view of the $W_{\mathfrak{s}}$-equivariant polar decomposition $(9), T_{\mathfrak{s}} / / W_{\mathfrak{s}}$ is a natural way the complexification of its compact real form $T_{\mathfrak{s}, \text { un }} / / W_{\mathfrak{s}}$. Similarly $\operatorname{Irr}^{\mathfrak{s}}(G)$ can be regarded as the "complexification" of $\operatorname{Irr}_{\mathrm{t}}(G)^{\mathfrak{s}}$ ABPS2, §2]. If we manage to construct a bijection (10) with suitable properties, then the method of [ABPS2, §4] shows that it extends to a bijection $\operatorname{Irr}(G)^{\mathfrak{s}} \longleftrightarrow T_{\mathfrak{s}} / / W_{\mathfrak{s}}$ with the same properties. Thus it suffices to prove the ABPS conjecture for tempered representations.

\section{The local Langlands correspondence}

4.1. Enhanced Langlands parameters. Let $W_{F}$ be the Weil group of $F$. We write $W_{F}^{\prime}:=W_{F} \times \mathrm{SL}_{2}(\mathbb{C})$ (that may be viewed as version of the Weil-Deligne group of $F$ ). Let $\Phi(G)$ denote the set of $G^{\vee}$-orbits of Langlands parameters for $G$, where $G^{\vee}$ is the Langlands dual group of $G$ (a complex Lie group with root datum dual to that of $G$ ).

The local Langlands conjecture (LLC) asserts notably that the set $\operatorname{Irr}(G)$ of isomorphism classes of irreducible smooth representations of $G$ can be parametrized by $\Phi(G)$. This parametrization is not a bijection in general. In fact, it is conjectured that each conjugacy class $\phi \in \Phi(G)$ is associated with a finite set $\Pi_{\phi}(G)$ of isomorphism classes of irreducible smooth representations of $G$, and they give a disjoint decomposition of $\operatorname{Irr}(G)$ :

$$
\operatorname{Irr}(G)=\bigsqcup_{\phi \in \Phi(G)} \Pi_{\phi}(G) .
$$

Such finite sets are called $L$-packets for $G$. Recent developments of the LLC can be found in $[\mathrm{Au}]$ and the references there.

In order to parametrize the irreducible representations in a given $L$-packet, we need more information than just the Langlands parameter itself.

We denote by $\mathrm{Z}^{\vee}$ the center of $G^{\vee}$. Let $G_{\mathrm{ad}}^{\vee}$ be the adjoint group of $G^{\vee}$, and let $G_{\mathrm{sc}}^{\vee}$ be the simply connected cover of the derived group of $G^{\vee}$.

Let $\phi: W_{F}^{\prime} \rightarrow G^{\vee}$ be an $L$-parameter. Let $\mathrm{Z}_{G_{\mathrm{ad}}^{\vee}}\left(\phi\left(W_{F}\right)\right)$ be the centralizer of $\phi\left(W_{F}\right)$ in $G_{\text {ad }}^{\vee}$, and let

$$
\mathcal{G}_{\phi}:=\mathrm{Z}_{G_{\mathrm{sc}}^{\vee}}^{1}\left(\phi\left(W_{F}\right)\right)
$$

be the inverse image of $\mathrm{Z}_{G_{\mathrm{ad}}}\left(\phi\left(W_{F}\right)\right)$ in $G_{\mathrm{sc}}^{\vee}$. We set $\overline{\mathcal{G}}_{\phi}:=\mathrm{Z}_{G^{\vee}}\left(\phi\left(W_{F}\right)\right)$. 
ExAmPLE 4.1. The group $\mathrm{Z}^{\vee}$ is $\{ \pm 1\}$ if $G=\mathrm{SO}_{2 n+1}(F)$, and is trivial if $G=\operatorname{Sp}_{2 n}(F)$. We have

$$
\begin{cases}\mathcal{G}_{\phi}=\overline{\mathcal{G}}_{\phi}=\mathrm{Z}_{\mathrm{Sp}_{2 n}(\mathbb{C})}\left(\phi\left(W_{F}\right)\right), & \text { if } G=\mathrm{SO}_{2 n+1}(F), \\ \mathcal{G}_{\phi}=\mathrm{Z}_{\operatorname{Spin}_{2 n+1}(\mathbb{C})}^{1}\left(\phi\left(W_{F}\right)\right) \text { and } \overline{\mathcal{G}}_{\phi}=\mathrm{Z}_{\mathrm{SO}_{2 n+1}(\mathbb{C})}\left(\phi\left(W_{F}\right)\right), & \text { if } G=\operatorname{Sp}_{2 n}(F) .\end{cases}
$$

We define the finite groups

$$
\mathcal{S}_{\phi}:=\pi_{0}\left(\mathrm{Z}_{G_{\mathrm{sc}}^{\vee}}^{1}(\phi)\right) \text { and } A_{\phi}:=\pi_{0}\left(\mathrm{Z}_{G^{\vee}}(\phi)\right) .
$$

We call any irreducible representation of $\mathcal{S}_{\phi}$ an enhancement of $\phi$. An irreducible representation $\rho$ of $\mathcal{S}_{\phi}$ is said to be $G$-relevant if its restriction to $\mathrm{Z}\left(G_{\mathrm{sc}}^{\vee}\right)^{W_{F}}$ is a multiple of the character which corresponds to the cohomology class defined by $G$ via the Kottwitz isomorphism

$$
X^{*}\left(\mathrm{Z}\left(G_{\mathrm{ad}}^{\vee}\right)^{W_{F}}\right) \simeq \mathrm{H}_{\mathrm{c}}^{1}\left(F, G_{\mathrm{ad}}\right) .
$$

An enhanced Langlands parameter (or enhanced $L$-parameter) for $G$ is a pair $(\phi, \rho)$, where $\phi \in \Phi(G)$ and $\rho$ is a $G$-relevant irreducible representation of the group $\mathcal{S}_{\phi}$. The group $\mathcal{S}_{\phi}$ coincides with the group considered by both Arthur in $\mathrm{Ar}$ and Kaletha in [Kal, §4.6]. We set

$$
u_{\phi}:=\phi\left(1,\left(\begin{array}{ll}
1 & 1 \\
0 & 1
\end{array}\right)\right),
$$

and denote $\mathcal{O}_{\phi}$ the conjugacy class of $u_{\phi}$ in $\mathcal{G}_{\phi}$.

The group $\mathcal{S}_{\phi}$ and $A_{\phi}$ are isomorphic to the groups

$$
A_{\mathcal{G}_{\phi}}\left(u_{\phi}\right):=\pi_{0}\left(\mathrm{Z}_{\mathcal{G}_{\phi}}^{1}\left(u_{\phi}\right)\right) \quad \text { and } \quad A_{\overline{\mathcal{G}}_{\phi}}\left(u_{\phi}\right):=\pi_{0}\left(\mathrm{Z}_{\overline{\mathcal{G}}_{\phi}}\left(u_{\phi}\right)\right) \text {, }
$$

respectively, see [AMS1, (92)] and [Mou1, §3].

Let $\mathcal{R}_{\phi}$ denote the component group of $\mathrm{Z}_{G^{\vee}}(\phi) / \mathrm{Z}^{\vee}$. It is expected that $\Pi_{\phi}(G)$ is in bijection with $\operatorname{Irr}\left(\mathcal{R}_{\phi}\right)$. The map $G_{\mathrm{sc}}^{\vee} \rightarrow G_{\text {ad }}^{\vee}$ induces a homomorphism $\mathcal{S}_{\phi} \rightarrow \mathcal{R}_{\phi}$. Set

$$
\mathcal{Z}_{\phi}:=\mathrm{Z}\left(G_{\mathrm{sc}}^{\vee}\right) / \mathrm{Z}\left(G_{\mathrm{sc}}^{\vee}\right) \cap \mathrm{Z}_{G_{\mathrm{sc}}^{\vee}}(\phi)^{\circ} .
$$

Then $\mathcal{S}_{\phi}$ is a central extension of $\mathcal{R}_{\phi}$ by $\mathcal{Z}_{\phi}$ (see [ABPS8, Lemma 1.7]):

$$
1 \rightarrow \mathcal{Z}_{\phi} \rightarrow \mathcal{S}_{\phi} \rightarrow \mathcal{R}_{\phi} \rightarrow 1
$$

Since $G_{\mathrm{sc}}^{\vee}$ is a central extension of $G_{\mathrm{ad}}^{\vee}=G^{\vee} / \mathrm{Z}\left(G^{\vee}\right)$, the conjugation action of $G_{\mathrm{sc}}^{\vee}$ on itself and on $\mathcal{S}_{\phi}$ descends to an action of $G_{\text {ad }}^{\vee}$. Via the canonical quotient map, also $G^{\vee}$ acts on $\mathcal{S}_{\phi}$ by conjugation.

We let $G^{\vee}$ act on the set of enhanced $L$-parameters for $G$ by

$$
g \cdot(\phi, \rho)=\left(g \phi g^{-1}, g \cdot \rho\right) \quad \text { where } \quad(g \cdot \rho)(a)=\rho\left(g^{-1} a g\right) .
$$

We denote by $\Phi_{\mathrm{e}}(G)$ the set of $G^{\vee}$-conjugacy classes of enhanced Langlands parameters for $G$.

4.2. The ordinary and generalized Springer correspondences. Let $\mathcal{G}$ be a complex (possibly disconnected) reductive group. Let $\mathcal{G}^{\circ}$ be its identity component. We denote by $\mathrm{U}(\mathcal{G})$ the unipotent variety of $\mathcal{G}$.

For $u \in \mathcal{G}$ unipotent, we denote by $A_{\mathcal{G}}(u)$ the component group of the centralizer of $u$ in $\mathcal{G}$, and by $\operatorname{Irr}\left(A_{\mathcal{G}}(u)\right)$ the set of equivalence classes of irreducible representations of $A_{\mathcal{G}}(u)$. 
Definition 4.2. The enhancement of $\mathrm{U}(\mathcal{G})$ is the set $\mathrm{U}_{\mathrm{e}}(\mathcal{G})$ of $\mathcal{G}$-conjugacy classes of pairs $(\mathcal{O}, \rho)$, with $\mathcal{O}$ is a unipotent class in $\mathcal{G}$, and $\rho \in \operatorname{Irr}\left(A_{\mathcal{G}}(u)\right)$, with $u \in \mathcal{O}$. The pair $(\mathcal{O}, \rho)$ is called an enhanced unipotent class in $\mathcal{G}$.

For $u$ a given unipotent element in $\mathcal{G}^{\circ}$, let $A_{\mathcal{G}}{ }^{\circ}(u)$ denote the component group of its centralizer $\mathrm{Z}_{\mathcal{G}} \circ(u)$ of $u$ in $\mathcal{G}^{\circ}$. Let $\mathcal{O}:=(u)_{\mathcal{G}}$ be the $\mathcal{G}^{\circ}$-conjugacy class of $u$. We denote by $\rho \mapsto \mathcal{E}_{\rho}$ the bijection between $\operatorname{Irr}\left(A_{\mathcal{G}} \circ(u)\right)$ and the irreducible $\mathcal{G}^{\circ}$-equivariant local systems $\mathcal{E}$ on $\mathcal{O}$, and by $\mathcal{E} \mapsto \rho_{\mathcal{E}}$ the inverse bijection. A representation $\rho^{\circ} \in \operatorname{Irr}\left(A_{\mathcal{G}} \circ(u)\right)$ is said to be cuspidal if the perverse sheaf $\operatorname{IC}\left(\mathcal{O}, \mathcal{E}_{\rho^{\circ}}\right)$ is cuspidal. Then, a representation $\rho \in \operatorname{Irr}\left(A_{\mathcal{G}}(u)\right)$ is cuspidal if its restriction to $A_{\mathcal{G}} \circ(u)$ is a direct sum of cuspidal irreducible representations of $A_{\mathcal{G}^{\circ}}(u)$ and an enhanced unipotent class $(\mathcal{O}, \rho)\left(\right.$ resp. $\left.\left(\mathcal{O}, \rho^{\circ}\right)\right)$ is called cuspidal if $\rho$ (resp. $\left.\rho^{\circ}\right)$ is cuspidal. We set

$$
\operatorname{Irr}_{\text {cusp }}\left(A_{\mathcal{G}}(u)\right):=\left\{\rho \in \operatorname{Irr}\left(A_{\mathcal{G}}(u)\right) \text { such that } \rho \text { is cuspidal }\right\} .
$$

A quasi-Levi subgroup of $\mathcal{G}$ is a subgroup $\mathcal{M}$ of the form $\mathcal{M}=\mathrm{Z}_{\mathcal{G}}\left(\mathrm{Z}(\mathcal{L})^{\circ}\right)$, with $\mathcal{L}$ a Levi subgroup of $\mathcal{G}^{\circ}$. The group $\mathcal{M}$ is said to be cuspidal if there exists a cuspidal enhanced unipotent pair in $\mathcal{M}$. Let $\mathfrak{B}\left(\mathrm{U}_{\mathrm{e}}(\mathcal{G})\right)$ be the set of $\mathcal{G}$-conjugacy classes of pairs $(\mathcal{M},(\mathcal{O}, \varepsilon))$, where $\mathcal{M}$ is a cuspidal quasi-Levi subgroup of $\mathcal{G}$, and $(\mathcal{O}, \varepsilon)$ is a cuspidal enhanced unipotent pair in $\mathcal{M}$.

The simple objects in $\operatorname{Perv}_{\mathcal{G}^{\circ}}\left(\mathrm{U}\left(\mathcal{G}^{\circ}\right)\right)$ are the $\operatorname{IC}(\mathcal{O}, \mathcal{E})$, where $\mathcal{O}$ is a unipotent class in $\mathcal{G}^{\circ}$ and $\mathcal{E}$ is an irreducible $\mathcal{G}^{\circ}$-equivariant $\overline{\mathbb{Q}}_{\ell}$-local system on $\mathcal{O}$. Let $(\mathcal{O}, \rho) \in \mathrm{U}_{\mathrm{e}}\left(\mathcal{G}^{\circ}\right)$ be an arbitrary enhanced unipotent class, and set $\mathcal{F}_{\rho}:=\operatorname{IC}\left(\mathcal{O}, \mathcal{E}_{\rho}\right)$. Then $\mathcal{F}_{\rho}$ occurs as a summand of $\mathrm{i}_{\mathcal{L}^{\circ} \subset \mathcal{P}}\left(\operatorname{IC}\left(\mathcal{O}_{0}, \mathcal{E}_{0}\right)\right)$, for some quadruple $\left(\mathcal{P}^{\circ}, \mathcal{M}^{\circ}, \mathcal{O}_{0}, \mathcal{E}_{0}\right)$, where $\mathcal{P}^{\circ}$ is a parabolic subgroup of $\mathcal{G}^{\circ}$ with Levi subgroup $\mathcal{M}^{\circ}$ and $\left(\mathcal{O}_{0}, \mathcal{E}_{0}\right)$ is a cuspidal enhanced unipotent class in $\mathcal{M}^{\circ}$ (see [Lus, §6.2] and [AHJR, Cor. 2.7]) and, moreover, $\left(\mathcal{P}^{\circ}, \mathcal{M}^{\circ}, \mathcal{O}_{0}, \mathcal{E}_{0}\right)$ is unique up to $\mathcal{G}^{\circ}$-conjugation (see [Lus, Prop. 6.3]). We denote by $\mathfrak{t}^{\circ}:=\left(\mathcal{M}^{\circ},\left(\mathcal{O}_{0}, \varepsilon_{0}\right)\right)_{\mathcal{G}^{\circ}}$ the $\mathcal{G}^{\circ}$-conjugacy class of $\left(\mathcal{M}^{\circ},\left(\mathcal{O}_{0}, \varepsilon_{0}\right)\right)$, where $\varepsilon_{0}$ corresponds to $\mathcal{E}_{0}$, and we call it the cuspidal support of the enhanced unipotent class $(\mathcal{O}, \rho)$.

The center $\mathrm{Z}\left(\mathcal{G}^{\circ}\right)$ of $\mathcal{G}^{\circ}$ maps naturally to $A_{\mathcal{G}^{\circ}}(\mathcal{O})$ and to $A_{\mathcal{M}^{\circ}}\left(\mathcal{O}_{0}\right)$. By construction [Lus, Theorem 6.5.a] $\rho$ and $\varepsilon_{0}$ have the same $\mathrm{Z}\left(\mathcal{G}^{\circ}\right)$-character. We define a cuspidal support map from

$$
\Psi_{\mathcal{G}^{\circ}}: \mathrm{U}_{\mathrm{e}}\left(\mathcal{G}^{\circ}\right) \rightarrow \mathfrak{B}\left(\mathrm{U}_{\mathrm{e}}\left(\mathcal{G}^{\circ}\right)\right)
$$

by sending the $\mathcal{G}^{\circ}$-conjugacy class of $(\mathcal{O}, \rho)$ to its cuspidal support $\mathfrak{t}^{\circ}=\left(\mathcal{M}^{\circ},\left(\mathcal{O}_{0}, \varepsilon_{0}\right)\right)_{\mathcal{G}^{\circ}}$.

Let $\mathfrak{t}^{\circ} \in \mathfrak{B}\left(\mathrm{U}_{\mathrm{e}}\left(\mathcal{G}^{\circ}\right)\right)$.

1. We denote by $\mathrm{U}_{\mathrm{e}}\left(\mathcal{G}^{\circ}\right)^{\mathfrak{t}^{\circ}}$ the fiber of $\mathfrak{t}$ under the map $\Psi_{\mathcal{G}^{\circ}}$.

2. Let $W_{\mathcal{M}^{\circ}}:=\mathrm{N}_{\mathcal{G}^{\circ}}\left(\mathcal{M}^{\circ}\right) / \mathcal{M}^{\circ}$, and let $W_{\mathfrak{t}^{\circ}}:=\mathrm{N}_{\mathcal{G}^{\circ}}\left(\mathfrak{t}^{\circ}\right) / \mathcal{M}^{\circ}$.

The group $W_{\mathcal{M}^{\circ}}$ is a Weyl group, it coincides with $W_{\mathfrak{t}}$ for every $\mathfrak{t}^{\circ} \in \mathfrak{B}\left(\mathrm{U}_{\mathrm{e}}\left(\mathcal{G}^{\circ}\right)\right)$, and

$$
\mathrm{U}_{\mathrm{e}}\left(\mathcal{G}^{\circ}\right)=\bigsqcup_{\mathfrak{t}^{\circ} \in \mathfrak{B}\left(\mathrm{U}_{\mathrm{e}}\left(\mathcal{G}^{\circ}\right)\right)} \mathrm{U}_{\mathrm{e}}\left(\mathcal{G}^{\circ}\right)^{\mathfrak{t}^{\circ}},
$$

and $\mathrm{U}_{\mathrm{e}}\left(\mathcal{G}^{\circ}\right)^{\mathfrak{t}^{\circ}}$ is in bijection with $\operatorname{Irr}\left(W_{\mathcal{M}^{\circ}}\right)$ (see [Lus] $)$. 
In AMS1 we extend the generalized Springer correspondence from $\mathcal{G}^{\circ}$ to $\mathcal{G}$ in the following way. Let $\mathfrak{t}=\left(\mathcal{M},\left(\mathcal{O}_{0}, \varepsilon\right)\right)_{\mathcal{G}} \in \mathfrak{B}\left(\mathrm{U}_{\mathrm{e}}(\mathcal{G})\right)$. We set

$$
W_{\mathfrak{t}}:=\mathrm{N}_{\mathcal{G}}(\tau) / \mathcal{M} \text { and } W_{\mathfrak{t}}^{\circ}:=\mathrm{N}_{\mathcal{G}}\left(\mathcal{M}^{\circ}\right) / \mathcal{M}^{\circ} \text {. }
$$

Let $(\mathcal{O}, \rho) \in \mathrm{U}_{\mathrm{e}}(\mathcal{G})$. There exists a map, called the cuspidal support map for $\mathrm{U}_{\mathrm{e}}(\mathcal{G})$

$$
\Psi_{\mathcal{G}}: \mathrm{U}_{\mathrm{e}}(\mathcal{G}) \rightarrow \mathfrak{B}\left(\mathrm{U}_{\mathrm{e}}(\mathcal{G})\right),
$$

which coincides to the map $\Psi_{\mathcal{G}}$ 。 from $(18)$ in the case when $\mathcal{G}$ is connected, such that

$$
\mathrm{U}_{\mathrm{e}}(\mathcal{G})=\bigsqcup_{\mathfrak{t} \in \mathfrak{B}\left(\mathrm{U}_{\mathrm{e}}(\mathcal{G})\right)} \mathrm{U}_{\mathrm{e}}(\mathcal{G})^{\mathfrak{t}},
$$

in which the fiber $\mathrm{U}_{\mathrm{e}}(\mathcal{G})^{\mathfrak{t}}$ of $\mathfrak{t}$ under the map $\Psi_{\mathcal{G}}$ is isomorphic to the group algebra $\mathbb{C}\left[W_{\mathfrak{t}}\right]$ of the finite group $W_{\mathrm{t}}$.

REMARK 4.3. When $\mathcal{G}$ is disconnected, the cocycle $\kappa_{\mathrm{t}}$ is not always trivial. It is trivial in the case when $\mathcal{M}^{\circ}$ is a torus as proved in ABPS6.

Definition 4.4. An enhanced $L$-parameter $(\phi, \rho) \in \Phi(G)_{\mathrm{e}}$ is called cuspidal if $\phi$ is discrete and $\left(u_{\phi}, \rho\right)$ is a cuspidal enhanced unipotent class in the group $\mathcal{G}_{\phi}$ defined in 12 . Cuspidality Conjecture 4.5 ([AMS1, §6]). The cuspidal enhanced Langlands parameters for $G$ correspond by the LLC to the irreducible supercuspidal representations of $G$.

4.3. Bernstein series of enhanced Langlands parameters. Applying the above construction to the group $\mathcal{G}=\mathcal{G}_{\phi}$, for every $(\phi, \rho) \in \Phi_{\mathrm{e}}(G)$, allows us to obtain a partition of the set of enhanced Langlands parameters into "Bernstein series".

Definition 4.6. Let $L$ be a Levi subgroup of $G$. An enhanced Langlands parameter $(\varphi, \varepsilon)$ for $L$ is cuspidal if $\varphi: W_{F}^{\prime} \rightarrow L^{\vee}$ is discrete and $\left(\mathcal{O}_{\varphi}, \varepsilon\right)$ is a cuspidal pair in $\mathcal{L}_{\phi}$, where $\mathcal{O}_{\varphi}$ is the $L^{\vee}$-conjugacy class of the unipotent element $\varphi\left(1,\left(\begin{array}{ll}1 & 1 \\ 0 & 1\end{array}\right)\right)$, and $\mathcal{L}_{\varphi}$ is defined analogously as $\mathcal{G}_{\phi}$, that is,

$$
\mathcal{L}_{\varphi}:=\mathrm{Z}_{L_{\mathrm{sc}}^{\vee}}^{1}\left(\varphi\left(W_{F}\right)\right)
$$

We will denote by $\Phi_{\mathrm{e}}(L)$ the set of $L^{\vee}$-orbits and by $\Phi_{\mathrm{e}, \mathrm{cusp}}(L)$ the subset of cuspidal $L^{\vee}$-orbits.

Let $(\phi, \rho) \in \Phi(G)$. Recall the unipotent element $u_{\phi}$ in $\mathcal{G}_{\phi}$ from $(12)$. We set

$$
\left[\mathcal{M}_{\phi},\left(\mathcal{O}_{0}, \varepsilon\right)\right]_{\mathcal{G}_{\phi}}:=\Psi_{\mathcal{G}_{\phi}}\left(u_{\phi}, \rho\right) \in \mathfrak{B}\left(\mathrm{U}_{\mathrm{e}}\left(\mathcal{G}_{\phi}\right)\right),
$$

where $\Psi_{\mathcal{G}_{\phi}}$ is the cuspidal support map for $\mathrm{U}_{\mathrm{e}}\left(\mathcal{G}_{\phi}\right)$ defined in (19). Then the cuspidal support of $(\phi, \rho)$ is defined to be

$$
\operatorname{Sc}(\phi, \rho):=\left(\mathrm{Z}_{G^{\vee}}\left(\mathrm{Z}\left(\mathcal{M}_{\phi}\right)^{\circ}\right), \varphi, \varepsilon\right) .
$$

On the other hand, the group of unramified characters of $L$ is naturally isomorphic to

$$
X_{\mathrm{nr}}\left({ }^{L} L\right):=\left\{\xi: W_{F} / I_{F} \rightarrow \mathrm{Z}_{G^{\vee}}^{\circ}\right\} .
$$

Given $(\varphi, \varepsilon) \in \Phi_{\mathrm{e}}(L)$, and $\xi \in X_{\mathrm{nr}}\left(L^{\vee}\right)$, define $(\xi \varphi, \varrho) \in \Phi_{\mathrm{e}}(L)$ by

$$
\xi \cdot \varphi(w, x):= \begin{cases}\varphi(w, t), & \text { if }(w, x) \in I_{F} \times \mathrm{SL}_{2}(\mathbb{C}), \\ \tilde{\xi} \varphi\left(\operatorname{Fr}_{F}\right), & \text { if }(w, x)=\left(\operatorname{Fr}_{F}, 1\right),\end{cases}
$$

where $\tilde{\xi} \in \mathrm{Z}_{L^{\vee} \rtimes I_{F}}^{\circ}$ represents $\xi$. 
Definition 4.7. We denote by $\mathfrak{s}^{\vee}:=\mathfrak{s}_{G}^{\vee}$ the $G^{\vee}$-conjugacy class of $\left(L^{\vee}, X_{\mathrm{nr}}\left(L^{\vee}\right) \cdot(\varphi, \varepsilon)\right)$, where $L$ is a Levi subgroup of $G$, and $(\varphi, \varepsilon)$ is a cuspidal enhanced Langlands parameter for $L$. We write

$$
\mathfrak{s}^{\vee}=\left[L^{\vee},(\varphi, \varepsilon)\right]_{G^{\vee}} .
$$

We call $\mathfrak{s}^{\vee}$ an inertial class for $\Phi_{\mathrm{e}}(G)$ and denote by $\mathfrak{B}^{\vee}(G)$ the set of such $\mathfrak{s}^{\vee}$.

Let $\mathfrak{s}^{\vee}=\left[L^{\vee},(\varphi, \varepsilon)\right]_{G^{\vee}} \in \mathfrak{B}^{\vee}(G)$. We set

$$
T_{\mathfrak{s}^{\vee}}:=\left\{(\xi \cdot \varphi)_{L^{\vee}}: \xi \in X_{\mathrm{nr}}\left({ }^{L} L\right)\right\} \simeq X_{\mathrm{nr}}\left({ }^{L} L\right) / X_{\mathrm{nr}}\left({ }^{L} L\right)(\varphi),
$$

where

$$
X_{\mathrm{nr}}\left({ }^{L} L\right)(\varphi):=\left\{\xi \in X_{\mathrm{nr}}\left({ }^{L} L\right):(\xi \cdot \varphi)_{L^{\vee}} \simeq(\varphi)_{L^{\vee}}\right\} .
$$

The following statement formulates a extension of Conjecture 4.5 to $\operatorname{Irr}(G)$ :

ConjeCture 4.8. Let $\mathfrak{s}=[L, \sigma]_{G} \in \mathfrak{B}(G)$. The LLC for $L$ induces a bijection

$$
\begin{aligned}
c: \mathfrak{B}(G) & \rightarrow \mathfrak{B}^{\vee}(G) \\
\mathfrak{s} & \mapsto \mathfrak{s}^{\vee},
\end{aligned}
$$

that satisfies the following properties:

(a) $T_{\mathfrak{s}} \simeq T_{\mathfrak{s} \vee}$

(b) $W_{\mathfrak{s}} \simeq W_{\mathfrak{s} \vee}$.

Conjecture 4.8 is known to be true in particular for split classical groups over a $p$-adic field of characteristic 0 Mou2 and for representations with unipotent reduction of an arbitrary $p$-adic group Sol3.

The construction that we will recall below was achieved in [AMS1] and leads to a decomposition

$$
\Phi_{\mathrm{e}}(G)=\bigsqcup_{\mathfrak{s}^{\vee} \in \mathfrak{B}^{\vee}(G)} \Phi_{\mathrm{e}}(G)^{\mathfrak{s}^{\vee}}
$$

Moreover, for each $\mathfrak{s}^{\vee}$ in $\mathfrak{B}^{\vee}(G)$, the results of AMS2 and AMS3 show that the subset $\Phi_{\mathrm{e}}(G)^{\mathfrak{s}^{\vee}}$ is in bijection with the simple modules of a generalized affine Hecke algebras with possibly unequal parameters.

Let $\mathfrak{s}^{\vee}=\left[{ }^{L} L,(\varphi, \varepsilon)\right]_{G^{\vee}} \in \mathfrak{B}^{\vee}(G)$. We associate to $\mathfrak{s}^{\vee}$ the finite group

$$
W_{\mathfrak{s}^{\vee}}:=\text { stabilizer of } \mathfrak{s}_{L^{\vee}}^{\vee} \text { in } \mathrm{N}_{G^{\vee}}\left(L^{\vee}\right) / L^{\vee} \text {. }
$$

Let $(\phi, \rho) \in \Phi_{\mathrm{e}}(G)^{\mathfrak{s}^{\vee}}$ and write as above $\Psi_{\mathcal{G}_{\phi}}\left(u_{\phi}, \rho\right)=:\left[\mathcal{M}_{\phi}, v, \varepsilon\right]_{\mathcal{G}_{\phi}}$. Fix a cuspidal datum $(L, \varphi, \varepsilon)$ and set $v:=u_{\varphi} \in{ }^{L} L$. Let $(\phi, \rho)$ be an enhanced $L$-parameter for $G$. We set

$$
\left[\mathcal{M}_{\phi}, v, \varepsilon\right]_{\mathcal{G}_{\phi}}:=\Psi_{G}\left(u_{\phi}, \rho\right) \text {. }
$$

We set

$$
{ }^{L} \Psi(\phi, \rho):=\left(\mathrm{Z}_{G^{\vee} \rtimes W_{F}}\left(\mathrm{Z}(\mathcal{M})^{\circ}\right),\left.\phi\right|_{W_{F}}, v, \varepsilon\right),
$$

a cuspidal datum for ${ }^{L} G$. Fix a cuspidal datum $\left({ }^{L} L,\left.\phi\right|_{W_{F}}, v, \varepsilon\right)$ for ${ }^{L} G$, and write

$$
\mathfrak{t}:=\left[G \cap L_{c}, v, \varepsilon\right]_{G}, \quad \mathfrak{t}^{\circ}=\left[G^{\circ} \cap L_{c}, v, \varepsilon_{0}\right]_{G^{\circ}} .
$$

The next result may be considered to be a version of the generalized Springer correspondence for enhanced $L$-parameters instead of enhanced unipotent classes. 
Proposition 4.9 ([AMS1]).

(a) There is a bijection

$$
\begin{aligned}
{ }^{L} \Sigma_{\mathfrak{t}}:{ }^{L} \Psi^{-1}\left({ }^{L} L,\left.\phi\right|_{W_{F}}, v, \varepsilon\right) & \longleftrightarrow \operatorname{Irr}\left(\mathbb{C}\left[W_{\mathfrak{t}}, \kappa_{\mathfrak{t}}\right]\right) \\
(\phi, \rho) & \longmapsto \Sigma_{\mathfrak{t}}\left(u_{\phi}, \rho\right) \\
\left(\left.\phi\right|_{W_{F}}, \Sigma_{\mathfrak{t}}^{-1}(\tau)\right) & \longleftrightarrow \tau .
\end{aligned}
$$

(b) Recall that we have a canonical bijection $\Sigma_{\mathfrak{t}^{\circ}}$ between $\operatorname{Irr}_{\mathbb{C}}\left(W_{\mathfrak{t}^{\circ}}\right)$ and $\Psi_{\mathcal{G}^{\circ}}^{-1}\left(\mathfrak{t}^{\circ}\right) \subset$ $\mathrm{U}_{\mathrm{e}}\left(\mathcal{G}^{\circ}\right)$. It relates to part (a) by

$$
\left.{ }^{L} \Sigma_{\mathfrak{t}}(\phi, \rho)\right|_{W_{\mathfrak{t}}}=\bigoplus_{i} \Sigma_{\mathfrak{t}^{\circ}}\left(u_{\phi}, \rho_{i}\right),
$$

where $\rho=\bigoplus_{i} \rho_{i}$ is a decomposition into irreducible $A_{\mathcal{G}}\left(u_{\phi}\right)$-subrepresentations.

(c) The $G^{\vee}$-conjugacy class of $\left(\left.\phi\right|_{W_{F}}, u_{\phi}, \rho_{i}\right)$ is determined by any irreducible $\mathbb{C}\left[W_{\mathbf{t}^{\circ}}\right]$ subrepresentation of ${ }^{L} \Sigma_{\mathfrak{t}}(\phi, \rho)$.

5. A Galois version of ABPS. Let $L$ be a Levi subgroup of $G$ and let $\phi_{L}: W_{F}^{\prime} \rightarrow L^{\vee}$ be a Langlands parameter for $L$.

Thanks to the decomposition 244, the following result reveals a new structure in the space of enhanced Langlands parameters for $G$, that of a disjoint union of extended quotients. It asserts that Conjecture 4 in [ABPS8] holds and is proved in [Mou2, Theorem 3.3] for $G$ a split classical group, and in [AMS1, Theorem 9.3] for general $G$.

THEOREM 5.1. Let $\mathfrak{s}_{L}^{\vee}=\left[L^{\vee},\left.\phi\right|_{W_{F}}, v, \varepsilon\right]_{L^{\vee}}$ be an inertial equivalence class for $L^{\vee}$. The maps ${ }^{L} \Sigma_{\mathfrak{t}}$ from Proposition 4.9 (a) combine to a bijection

$$
\begin{aligned}
\Phi_{\mathrm{e}}(G)^{\mathfrak{s}^{\vee}} & \longleftrightarrow \Phi_{\mathrm{e}}(L)^{\mathfrak{s}_{L}} / / W_{\mathfrak{s}^{\vee}} \\
(\phi, \rho) & \longmapsto\left({ }^{L} \Psi(\phi, \rho), \Sigma_{\mathfrak{t}}\left(u_{\phi}, \rho\right)\right) \\
\left(\left.\phi\right|_{W_{F}}, \Sigma_{\mathfrak{t}}^{-1}(\tau)\right) & \longleftrightarrow\left(L^{\vee},\left.\phi\right|_{W_{F}}, v, \varepsilon, \tau\right),
\end{aligned}
$$

with the following properties:

- it preserves boundedness of (enhanced) L-parameters,

- the restriction of $\tau$ to $W_{\mathfrak{t}^{\circ}}$ canonically determines the (non-enhanced) L-parameter in ${ }^{L} \Sigma_{\mathfrak{t}}(\tau)$,

6. Link with the Baum-Connes conjecture. This section is a simplified version of [ABPS8, Section 4] for split $p$-adic groups.

Let $X$ be a locally compact Hausdorff space and let $\Gamma$ be a group acting on $X$. For simplicity we assume that $\Gamma$ is finite. The $\Gamma$-equivariant $\mathrm{K}$-theory of $X$ was defined in [Ati §2.4]. When $X$ is compact, $K_{\Gamma}^{0}(X)$ is the Grothendieck group of the semigroup of complex $\Gamma$-vector bundles on $X$. When $X$ is only locally compact, we let $X \cup\{\infty\}$ be its one-point compactification, and we put

$$
K_{\Gamma}^{0}(X)=\operatorname{ker}\left(K_{\Gamma}^{0}(X \cup\{\infty\}) \rightarrow K_{\Gamma}^{0}(\{\infty\})\right) .
$$

We recall that the equivariant $K^{1}$-group is defined via the suspension functor. It can be expressed as

$$
K_{\Gamma}^{1}(X)=K_{\Gamma}^{0}(X \times \mathbb{R}),
$$


where $\Gamma$ acts trivially on $\mathbb{R}$. Typically one writes

$$
K_{\Gamma}^{*}(X)=K_{\Gamma}^{0}(X) \oplus K_{\Gamma}^{1}(X)
$$

a $\mathbb{Z} / 2 \mathbb{Z}$-graded abelian group. Let

$$
C_{0}(X)=\{f \in C(X \cup\{\infty\}, \mathbb{C}): f(\infty)=0\}
$$

be the commutative $C^{*}$-algebra of functions on $X$ which vanish at infinity. By the SerreSwan Theorem its K-theory is

$$
K_{*}\left(C_{0}(X)\right) \cong K^{*}(X) .
$$

The group $\Gamma$ acts on $C_{0}(X)$ by automorphisms, and we form the crossed product $C_{0}(X) \rtimes \Gamma$. We have $\operatorname{Irr}\left(C_{0}(X) \rtimes \Gamma\right) \cong X / / \Gamma$ (see [ABPS6]). By the Green-Julg Theorem [Jul] and the equivariant Serre-Swan Theorem [Phi, Theorem 2.3.1] there is a natural isomorphism

$$
K_{*}\left(C_{0}(X) \rtimes \Gamma\right) \cong K_{\Gamma}^{*}(X) .
$$

Thus we can interpret $K_{\Gamma}^{*}(X)$ as the K-theory of the topological space $X / / \Gamma$. That space being usually not Hausdorff, the statement is not precise, it is rather a manifestation of the philosophy of noncommutative geometry.

As before, let $G$ be a reductive $p$-adic group. Let $\mathcal{H}(G)$ denote the Hecke algebra of $G$, that is, the vector space $C_{\mathrm{c}}^{\infty}(G)$ of locally constant compactly supported functions on $G$, endowed with the convolution product. It is the version of the group algebra of $G$ which is most suitable for studying smooth representations. The category $\operatorname{Rep}(G)$ is naturally equivalent with the category $\operatorname{Rep}(\mathcal{H}(G))$ of $\mathcal{H}(G)$-modules $V$ such that $\mathcal{H}(G) \cdot V=V$.

The reduced $C^{*}$-algebra $C_{\text {red }}^{*}(G)$ is the completion of $\mathcal{H}(G)$ in the algebra of bounded linear operators on the Hilbert space $L^{2}(G)$. It follows from the work of Harish-Chandra (see [Vig, §10]) that the irreducible representations of $C_{\text {red }}^{*}(G)$ can be identified with those of the Schwartz algebra of $G$. By [Wal, §III.7] the latter are the same as irreducible tempered $G$-representations. Thus we get

$$
\operatorname{Irr}\left(C_{\text {red }}^{*}(G)\right)=\operatorname{Irr}_{\mathrm{t}}(G),
$$

which means that $C_{\text {red }}^{*}(G)$ is the correct $C^{*}$-algebra to study the noncommutative geometry of the tempered dual of $G$. The structure of $C_{\text {red }}^{*}(G)$ was described by means of the Fourier transform in $\mathrm{Ply}$.

The Baum-Connes conjecture provides a picture of the K-theory of this $C^{*}$-algebra in geometric terms. Let $\mathcal{I}(G)$ be the (nonreduced) Bruhat-Tits building of $G$. This is a proper $G$-space with many remarkable properties, for example:

- $\mathcal{I}(G)$ satisfies the negative curvature inequality [Tit, 2.3] and hence is contractible and has unique geodesics [Bro, §VI.3];

- every compact subgroup of $G$ fixes a point of $\mathcal{I}(G)$, see [Tit, §2.3.1] or [Bro, §VI.4].

In view of [ $\mathrm{BCH}$, Proposition 1.8], these properties make $\mathcal{I}(G)$ into a universal space for proper $G$-actions [BCH, Definition 1.6]. 
Let $K_{*}^{G}(\mathcal{I}(G))$ denote the $G$-equivariant K-homology of $\mathcal{I}(G)$ as defined in $\overline{\mathrm{KaS}}$. The Baum-Connes conjecture asserts that the canonical assembly map

$$
K_{*}^{G}(\mathcal{I}(G)) \rightarrow K_{*}\left(C_{\text {red }}^{*}(G)\right)
$$

is an isomorphism. This was proven (for a large class of groups containing $G$ ) in [Laf]. For the groups under consideration the Baum-Connes conjecture can also be formulated and proven more algebraically [HiNi], [Sch], with equivariant cosheaf homology (also known as chamber homology). By [Sol1] these two versions of the conjecture are compatible.

The left-hand side of 32 , defined in terms of $K$-cycles, has never been directly computed for a noncommutative reductive $p$-adic group. Results of Voigt [Voi] allow us to replace the left-hand side with the chamber homology groups. Chamber homology has been directly computed for only two noncommutative $p$-adic groups: $\mathrm{SL}_{2}(F)[\mathrm{BHP}$ and $\mathrm{GL}_{3}(F)$ AHP. In the case of $\mathrm{GL}_{3}(F)$, one can be sure that representative cycles in all the homology groups have been constructed only by checking with the right-hand side of the Baum-Connes conjecture. In other words, one always has to have an independent computation of the right-hand side.

On the $C^{*}$-algebra of $(32)$ side our earlier conjectures have something to say. The Bernstein decomposition of $\mathcal{H}(G)$ :

$$
\mathcal{H}(G)=\bigoplus_{\mathfrak{s} \in \mathfrak{B}(G)} \mathcal{H}(G)^{\mathfrak{s}}
$$

gives rise to a decomposition

$$
C_{\text {red }}^{*}(G)=\bigoplus_{\mathfrak{s} \in \mathfrak{B}(G)} C_{\text {red }}^{*}(G)^{\mathfrak{s}} \quad \text { with } \quad \operatorname{Irr}\left(C_{\text {red }}^{*}(G)^{\mathfrak{s}}\right)=\operatorname{Irr}_{\mathrm{t}}(G)^{\mathfrak{s}}
$$

Combining this with (6) leads to the following conjecture that is the specialization of Conjecture 5 of ABPS8] to the case where $G$ is split:

CONJECTURE 6.1. Let $\mathfrak{s} \in \mathfrak{B}(G)$. There exists a canonical isomorphism

$$
K_{W_{\mathfrak{s}}}^{*}\left(T_{\mathfrak{s}, \text { un }}\right) \rightarrow K_{*}\left(C_{\text {red }}^{*}(G)^{\mathfrak{s}}\right),
$$

where $K_{W_{\mathfrak{s}}}^{j}\left(T_{\mathfrak{s}, \text { un }}\right)$ is the classical topological equivariant $K$-theory for the group $W_{\mathfrak{s}}$ acting on the compact torus $T_{\mathfrak{s}, \text { un }}$ introduced in Definition 3.1 .

Conjecture 6.1 that can be viewed as the topological K-theory version of the ABPS conjecture, provides a much finer and more precise formula for $K_{*}\left(C_{\text {red }}^{*}(G)\right)$ than BaumConnes alone. In the case when $\mathfrak{s}=\left[L, \operatorname{triv}_{L}\right]_{G}$, with $L$ a torus, it is established modulo torsion in ABPS8, (4.9)]. In the more general case when $\mathfrak{s}=[L, \sigma]_{G}$ and the supercuspidal representation $\sigma$ of $L$ has unipotent reduction (that is, it is a depth-zero representation obtained from a unipotent cuspidal representation of the reductive quotient of a parahoric subgroup of $L$ ), then Conjecture 6.1 follows from the combination of [Sol2] and Sol3].

\section{References}

[AHJR] P. Achar, A. Henderson, D. Juteau, S. Riche, Modular generalized Springer correspondence I: the general linear group, J. Eur. Math. Soc. (JEMS) 18 (2016), 1405-1436. 
[Ar] J. Arthur, A note on L-packets, Pure Appl. Math. Q. 2 (2006), 199-217.

[Ati] M. F. Atiyah, K-theory, Math. Lecture Note Ser., W. A. Benjamin, New York, 1967.

[Au] A.-M. Aubert, Around the Langlands program, Jahresber. Dtsch. Math.-Ver. 120 (2018), 3-40.

[ABP1] A.-M. Aubert, P. Baum, R. J. Plymen, The Hecke algebra of a reductive p-adic group: a geometric conjecture, in: Noncommutative Geometry and Number Theory, Aspects of Math. E37, Vieweg, Wiesbaden, 2006, 1-34.

[ABP2] A.-M. Aubert, P. Baum, R. J. Plymen, Geometric structure in the representation theory of reductive p-adic groups II, in: Harmonic Analysis on Reductive, $p$-adic Groups, Contemp. Math. 543, Amer. Math. Soc., Providence, RI, 2011, 71-90.

[ABP3] A.-M. Aubert, P. F. Baum, R. J. Plymen, Geometric structure in the principal series of the p-adic group $\mathrm{G}_{2}$, Represent. Theory 15 (2011), 126-169.

[ABPS1] A.-M. Aubert, P. Baum, R. J. Plymen, M. Solleveld, Geometric structure in smooth dual and local Langlands correspondence, Japan. J. Math. 9 (2014), 99-136.

[ABPS2] A.-M. Aubert, P. Baum, R. J. Plymen, M. Solleveld, On the local Langlands correspondence for non-tempered representations, Münster J. Math. 7 (2014), 27-50.

[ABPS3] A.-M. Aubert, P. Baum, R. J. Plymen, M. Solleveld, Depth and the local Langlands correspondence, in: Arbeitstagung Bonn 2013, Progr. Math. 319, Birkhäuser/ Springer, Cham, 2016, 17-41.

[ABPS4] A.-M. Aubert, P. Baum, R. J. Plymen, M. Solleveld, The local Langlands correspondence for inner forms of $\mathrm{SL}_{n}$, Res. Math. Sci. 3 (2016), Paper No. 32.

[ABPS5] A.-M. Aubert, P. Baum, R.J. Plymen, M. Solleveld, Geometric structure for the principal series of a split reductive p-adic group with connected centre, J. Noncommut. Geom. 10 (2016), 663-680.

[ABPS6] A.-M. Aubert, P. Baum, R. J. Plymen, M. Solleveld, The principal series of p-adic groups with disconnected centre, Proc. Lond. Math. Soc. (3) 114 (2017), 798-854.

[ABPS7] A.-M. Aubert, P. Baum, R. J. Plymen, M. Solleveld, Hecke algebras for inner forms of p-adic special linear groups, J. Inst. Math. Jussieu 16 (2017), 351-419.

[ABPS8] A.-M. Aubert, P. Baum, R. J. Plymen, M. Solleveld, Conjectures about p-adic groups and their noncommutative geometry, in: Around Langlands Correspondences, Contemp. Math. 691, Amer. Math. Soc., Providence, RI, 2017, 15-51.

[ABPS9] A.-M. Aubert, P. Baum, R. J. Plymen, M. Solleveld, Smooth duals of inner forms of $\mathrm{GL}_{n}$ and $\mathrm{SL}_{n}$, Doc. Math. 24 (2019), 373-420.

[ABPS10] A.-M. Aubert, P. Baum, R. J. Plymen, M. Solleveld, Morita equivalences for $k$-algebras, this volume, 245-265.

[AHP] A.-M. Aubert, S. Hasan, R. J. Plymen, Cycles in the chamber homology of GL(3), K-Theory 37 (2006), 341-377.

[AMS1] A.-M. Aubert, A. Moussaoui, M. Solleveld, Generalizations of the Springer correspondence and cuspidal Langlands parameters, Manuscripta Math. 157 (2018), 121-192.

[AMS2] A.-M. Aubert, A. Moussaoui, M. Solleveld, Graded Hecke algebras for disconnected reductive groups, in: Geometric Aspects of the Trace Formula, Simons Symp., Springer, Cham, 2018, 23-84.

[AMS3] A.-M. Aubert, A. Moussaoui, M. Solleveld, Affine Hecke algebras for Langlands parameters, arXiv:1701.03593.

[APS] A.-M. Aubert, R. J. Plymen, M. Solleveld, Contribution to Celebratio Mathematica, https://celebratio.org/Baum_P/article/714/. 
[BCH] P. F. Baum, A. Connes, N. Higson, Classifying space for proper actions and K-theory of group $C^{*}$-algebras, in: $C^{*}$-algebras: 1943-1993. A fifty year celebration, Contemp. Math. 167, Amer. Math. Soc., Providence, RI, 1994, 240-291.

[BHP] P. Baum, N. Higson, R. J. Plymen, Equivariant homology for SL(2) of a p-adic field, in: Index Theory and Operator Algebras, Contemp. Math. 148, Amer. Math. Soc., Providence, RI, 1993, 1-18.

[BN] P. Baum, V. Nistor, Periodic cyclic homology of Iwahori-Hecke algebras, K-Theory 27 (2002), 329-357.

[BeDe] J. Bernstein, Le "centre" de Bernstein, in: Représentations des groupes réductifs sur un corps local, Travaux en cours, Hermann, Paris, 1984, 1-32.

[BrP] J. Brodzki, R. J. Plymen, Complex structure on the smooth dual of GL $(n)$, Doc. Math. 7 (2002), 91-112.

[Bro] K. S. Brown, Buildings, Springer, New York, 1989.

[HiNi] N. Higson, V. Nistor, Cyclic homology of totally disconnected groups acting on buildings, J. Funct. Anal. 141 (1996), 466-495.

[Jul] P. Julg, K-théorie équivariante et produits croisés, C. R. Acad. Sci. Paris Sér. I Math. 292 (1981), 629-632.

[Kal] T. Kaletha, Global rigid inner forms and multiplicities of discrete automorphic representations, Invent. Math. 213 (2018), 271-369.

[Kas] G. Kasparov, Equivariant KK-theory and the Novikov conjecture, Invent. Math. 91 (1988), 147-201.

[Laf] V. Lafforgue, K-théorie bivariante pour les algèbres de Banach et conjecture de BaumConnes, Invent. Math. 149 (2002), 1-95.

[Lus] G. Lusztig, Intersection cohomology complexes on a reductive group, Invent. Math. 75 (1984), 205-272.

[Mou1] A. Moussaoui, Centre de Bernstein dual pour les groupes classiques, Represent. Theory 21 (2017), 172-246.

[Mou2] A. Moussaoui, Proof of the Aubert-Baum-Plymen-Solleveld conjecture for split classical groups, in: Around Langlands Correspondences, Contemp. Math. 691, Amer. Math. Soc., Providence, RI, 2017, 257-281.

[Opd] E. M. Opdam, On the spectral decomposition of affine Hecke algebras, J. Inst. Math. Jussieu 3 (2004), 531-648.

[Phi] N. C. Phillips, Equivariant K-theory and freeness of group actions on $C^{*}$-algebras, Lecture Notes in Math. 1274, Springer, Berlin, 1987.

[Ply] R. J. Plymen, Reduced $C^{*}$-algebra for reductive p-adic groups, J. Funct. Anal. 88 (1990), 251-266.

[Sch] P. Schneider, Equivariant homology for totally disconnected groups, J. Algebra 203 (1998), 50-68.

[Sol1] M. Solleveld, On the classification of irreducible representations of affine Hecke algebras with unequal parameters, Represent. Theory 16 (2012), 1-87.

[Sol2] M. Solleveld, Topological K-theory of affine Hecke algebras, Ann. K-Theory 3 (2018), 395-460.

[Sol3] M. Solleveld, On unipotent representations of ramified p-adic groups, arXiv: 1912.08451

[Tit] J. Tits, Reductive groups over local fields, in: Automorphic Forms, Representations and L-functions. Part I, Proc. Sympos. Pure Math. 33, Amer. Math. Soc., Providence, RI, 1979, 29-69. 
[Vig] M.-F. Vignéras, On formal dimensions for reductive -adic groups, in: Festschrift in honor of I. I. Piatetski-Shapiro on the occasion of his sixtieth birthday, Part I, Israel Math. Conf. Proc. 2, Weizmann, Jerusalem, 1990, 225-266.

[Voi] C. Voigt, Chern character for totally disconnected groups, Math. Ann. 343 (2009), 507-540.

[Wal] J.-L. Waldspurger, La formule de Plancherel pour les groupes p-adiques (d'après Harish-Chandra), J. Inst. Math. Jussieu 2 (2003), 235-333. 